Japan. J. Med. Sci. Biol., 42, 13-23, 1989.

\title{
TOXOPLASMA GONDII: A SIMPLE METHOD FOR TITRATION OF INFECTIVITY WITH MONOLAYER CELLS
}

Takuro ENDOH and Kenji YAGITA

Department of Parasitology, National Institute of Health, Kamiosaki, Shinagawaku, Tokyo 141

(Received May 1, 1989. Accepted June 27, 1989)

SUMMARY: A simple method for assessing the infectivity of Toxoplasma gondii using primary cultured monolayer cells has been devised. Statistic analysis important for interpreting the results of such experimentation was made. In this method, the number of intracellular toxoplasmas and the percentage of confluency of the monolayer were measured. The latter value was used for conversion of the number of intracellular toxoplasmas per unit area to the percentage of infective toxoplasmas. A linear dose-response relationship between the number of intracellular toxoplasmas per unit area and that of toxoplasmas inoculated was demonstrated with primary cell monolayers from the lungs of two-day old inbred golden hamsters and the kidneys of newborn mice and newborn Wistar Imamichi strain rats. On the average, $37 \%$ of the toxoplasma organisms harvested from the peritoneal exudate of mice on the third or fourth day of infection were found to be infective. This value compares very favorably with the value $40 \%$ reported previously.

\section{INTRODUCTION}

Toxoplasma gondii, an obligate intracellular protozoan parasite, has a worldwide distribution and infects man and many species of mammals and birds. Despite intensive studies by experimental infection of various animals and tissue

遠藤卓郎·八木田健司(国立予防衛生研究所寄生虫部) 
cultures, relatively little is known about this parasite's requirements for infection, survival and growth. An appropriate method to titrate the infectivity of the parasite is a prerequisite for such investigations of the organism. Rate of entry has been most frequently expressed as percentage of infected cells. However, this simple enumeration of infected cells is incomplete in the evaluation of infectivity. Chaparas and Schlesinger (1) applied the plaque-counting method of Dulbecco and Vogt (2) for titration of the infectivity of $T$. gondii in cell cultures. A tissue-culture method for quantitative determination of parasite infectivity was also presented by Lycke and Lund (3) using HeLa cell cultures. Some modifications based on these methods have also been employed in various experiments (4-6); but, each has one or more shortcomings, such as a long incubation period among others.

In the present study an improved method for titration is presented using primary culture monolayer cells in which monolayer cells from various animals can be used as host cells.

\section{MATERIALS AND METHODS}

Parasite: The virulent strain ( $\mathrm{RH})$ of Toxoplasma gondii was maintained by serial intraperitoneal passages at 3- to 4-day intervals in mice. The trophozoites of $T$. gondii (toxoplasmas) were harvested from the peritoneal exudate of mice on the third or fourth day of infection. They were washed twice by centrifugation at 300 $\times \mathrm{g}$ in $\mathrm{K}_{2} \mathrm{SO}_{4}$-based isotonic solution, $\mathrm{pH} 8.2\left(\mathrm{~K}_{2} \mathrm{SO}_{4}\right.$-buffer) (7) and applied to a Millipore filter (12- $\mu \mathrm{m}$ pore size) to remove mouse peritoneal cells. The filtrate was centrifuged and resuspended in the same buffer at 108 cells $/ \mathrm{ml}$. The viability of the parasites was monitored by trypan blue dye exclusion.

Primary cell culture: Trypsin-dispersed cell suspensions were obtained from the lungs of two-day old inbred golden hamsters (the HAG strain; established and maintained at NIH of Japan). The cells were washed twice by centrifugation at $300 \times \mathrm{g}$ for 5 min with Eagle's minimum essential medium (MEM) containing $10 \%$ heat-inactivated fetal calf serum to remove trypsin, resuspended into the same medium at a concentration of approximately $106 \mathrm{cells} / \mathrm{ml}$ and plated onto a plastic culture dish. They were then cultured in a $\mathrm{CO}_{2}$ incubator for 4 days. Monolayer cells (BHL) collected after 4 days were again trypsinized, and subdivided for further serial subcultures at 4-day intervals. Similarly, primary cultures were prepared from the kidneys of new born C57BL/6J strain mice (BMK) and from the kidneys of newborn Wistar Imamichi strain rats (BRK). Some of these cells (within five passages) were cryopreserved.

Infection of monolayer cells by $\mathrm{T}$. gondii: Monolayers on coverslips were covered with aliquots of a suspension containing appropriate numbers of $T$. gondii 
$\left(2.5 \times 10^{7}\right.$ organisms $/ 5 \mathrm{ml} / 60-\mathrm{mm}$ culture dish) immediately after diluted into MEM containing $10 \mathrm{mM}$ Hepes, $\mathrm{pH} 7.5$. They were incubated for $30 \mathrm{~min}$ to allow infection, washed thoroughly with MEM to remove free parasites and reincubated for additional $2 \mathrm{hr}$. In our preliminary experiments, most of the toxoplasmas entered the host cells in as rapidly as $15 \mathrm{~min}$, and the number entering cells did not increase after $20 \mathrm{~min}$.

Assessment of toxoplasma infection: We used here primary cultures for the following reasons: [1] unlike cell-line cells, primary culture cells possess the feature of contact inhibition, so they do not overlap during multiplication. [2] They are active enough to form a thin film spreading widely over the cover slip. [3] Using these monolayers, we were able to observe stained specimens at high resolution under a light microscope.

The monolayers infected with toxoplasmas were fixed in absolute methanol and were stained with Giemsa solution. Some specimens were fixed $18 \mathrm{hr}$ after infection to confirm the parasite potential for multiplication in host cells. Observations were made by light microscopy for the number of intracellular toxoplasmas in $0.09 \mathrm{~mm}^{2}$ of the monolayer among at least a total 20 quadrants of duplicate cover-slip cultures. A grid-type optical micrometer, $10 \mathrm{~mm}$ on a side and graduated in tenths of a degree (making 100 squares), in a $10 \times$ optic covers 0.09 $\mathrm{mm}^{2}$ (1 Unit Area: UA) with a $40 \times$ objective. The percentage of confluency of the monolayer (percentage of the efficacious cellular area for toxoplasma infection) was measured by a semiautomatic image analysis system CB-SAIAS (Computer Bild, Tokyo). This value can also be calculated by weighing cut-outs of micrographs of the monolayers and those of cellular areas.

Media and reagent sources: The following reagents were purchased from Sigma; tris (hydroxymethyl) aminomethane (Tris), N-2-hydroxy ethylpiperazineN'-2-ethanesulfonic acid (Hepes). Tris-buffer was prepared by titrating with $\mathrm{H}_{2} \mathrm{SO}_{4}$ and Hepes buffer with $\mathrm{NaOH}$. Minimum essential medium (MEM) was purchased from Nissui Co (Tokyo). $\mathrm{K}_{2} \mathrm{SO}_{4}$-buffer has the following composition: $44.7 \mathrm{mM} \mathrm{K}_{2} \mathrm{SO}_{4}, 106 \mathrm{mM}$ sucrose, $10 \mathrm{mM} \mathrm{MgSO}_{4}, 20 \mathrm{mM}$ Tris- $\mathrm{H}_{2} \mathrm{SO}_{4}$ (pH 8.2), 5 $\mathrm{mM}$ glucose, and $3.5 \mathrm{mg} / \mathrm{ml}$ bovine serum albumine (BSA).

\section{RESULTS}

To develop a simple method to measure the infectivity of the trophozoites of Toxoplasma gondii, primary cell monolayers were incubated with toxoplasmas, and toxoplasmas/host cells/UA were counted in different quadrants of cover slip cultures. The cultures employed in the present experiments had not been cloned, and thus they consisted of different cell types, resulting in variation in the number of host cells/UA in different quadrants (Fig. 1). Toxoplasmas in $\mathrm{K}_{2} \mathrm{SO}_{4}$-buffer 


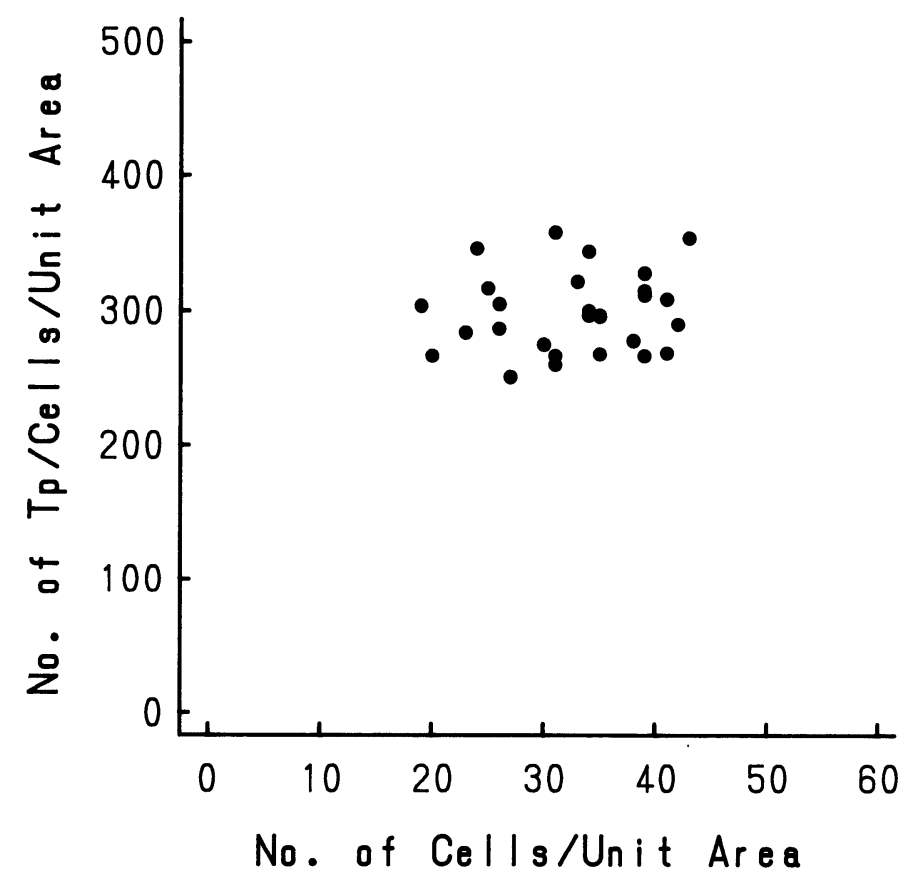

Fig. 1. Correlation between the number of intracellular Toxoplasma gondii/UA and that of host cells/UA. The numbers of intracellular toxoplasmas/UA were plotted against those of host cells/UA. Regression analysis indicated a slope of 0.546 , intercept $=281.0$ and $\gamma=0.127$, demonstrating no correlation between the two values.

containing $5 \mathrm{mM}$ glucose and $3.5 \mathrm{mg} / \mathrm{ml} \mathrm{BSA}, \mathrm{pH} 8.2$, consistently showed a high infectivity for monolayer cells when they were overlayed onto monolayers immediately after being resuspended in MEM, $\mathrm{pH}$ 7.5.

\section{Influence of Monolayer Cells/UA on the Number of Intracellular Toxoplasmas/UA}

First, confluent monolayers were infected with toxoplasmas at a constant inoculum size of the organisms. The number of intracellular toxoplasmas/UA and that of host cells/UA in different quadrants were recorded.

The relationship between the number of intracellular toxoplasmas/UA and that of host cells/UA is illustrated in Fig. 1. The number of cells/UA varied from 19 to 43 with the average of $32.1 \pm 6.9$, and out of approximately 796 toxoplasmas/UA (equivalent to $2.5 \times 10^{7}$ toxoplamas/60-mm culture dish) plated, $299.4 \pm 32.8$ toxoplasmas were found in host cells/UA. About $38 \%$ of the parasites inoculated 
Table I. Comparison of the number of intracellular Toxoplasma gondii per unit area to the percentage of cellular area

\begin{tabular}{lllll}
\hline & $\begin{array}{l}\text { Number of } \\
\text { intracellular } \\
\text { toxoplasmas/ } \\
\text { unit area }\end{array}$ & $\begin{array}{l}\text { Number of } \\
\text { host cells/ } \\
\text { unit area }\end{array}$ & $\begin{array}{l}\text { Degree of } \\
\text { confluency } \\
(\%)\end{array}$ & $\begin{array}{l}\text { Infective\# } \\
\text { toxoplasmas } \\
(\%)\end{array}$ \\
\hline Exp. I & $299.4 \pm 32.8$ & $32.1 \pm 6.9$ & 100.0 & 37.6 \\
Exp. II* & $279.8 \pm 25.8$ & $39.7 \pm 6.5$ & 96.3 & 36.5 \\
Exp. III & $264.0 \pm 33.4$ & $17.9 \pm 4.3$ & 88.6 & 37.4 \\
Exp. IV & $222.1 \pm 21.2$ & $18.8 \pm 4.1$ & 75.0 & 37.2 \\
Exp. V** & $206.3 \pm 21.7$ & $7.8 \pm 2.6$ & 73.2 & 35.4 \\
Exp. VI & $156.3 \pm 21.9$ & $21.0 \pm 6.1$ & 51.7 & 37.9 \\
Exp. VII & $146.6 \pm 15.6$ & $14.5 \pm 1.7$ & 48.4 & 38.1 \\
\hline
\end{tabular}

BHL cell monolayers at various degrees of confluency were infected with $2.5 \times 107$ toxoplasmas $/ 5 \mathrm{ml} / 60$ - $\mathrm{mm}$ culture dish. In some experiments, *BRK or **BMK cell monolayers were similarly infected with toxoplasmas. \#Percentage of infective toxoplasmas was calculated as follows:

Number of intracellular toxoplasmas/unit area $\times 100$

Number of inoculated toxoplasmas/unit area

Degree of confluency

were demonstrated to enter the host cells within $30 \mathrm{~min}$ of incubation at $37 \mathrm{C}$ (Table I). It was also demonstrated that increasing the number of host cells/UA did not influence the number of intracellular toxoplasmas/UA (Fig. 1). Regression analysis indicates a slope of 0.546 , intercept $=281.0$ and its correlation coefficient of $\gamma=0.1268$, demonstrating no significant correlation between two values. These results suggest strongly that when the cultures reached the confluent state, the monolayers provide a uniform surface for infection with each toxoplasma. 


\section{Relationship between the Number of Intracellular Toxoplasmas/UA and the Percentage of Confluency of Monolayer}

Secondly, BHL monolayers, at varying degree of confluency, were infected with toxoplasmas at a constant inoculum size. In some experiments, BRK and BMK monolayers were similarly infected with toxoplasmas. The percentage of confluency in each culture was recorded by a semiautomatic image analysis system CB-SAIAS. The number of intracellular toxoplasmas/UA increased with increasing percentage of confluency (Table I). The results presented in Fig. 2 demonstrate that the relationship between the number of intracellular toxoplasmas/UA and the percentage of confluency is linear. The correlation coefficient obtained was close to $1(\gamma=0.996)$. Consequently, the percentage of infective toxoplasmas calculated from the number of intracellular toxoplasmas/UA divided by the number of inoculated toxoplasmas/UA, multiplied by 100 , and divided by the percentage of confluency in various experiments was constant,

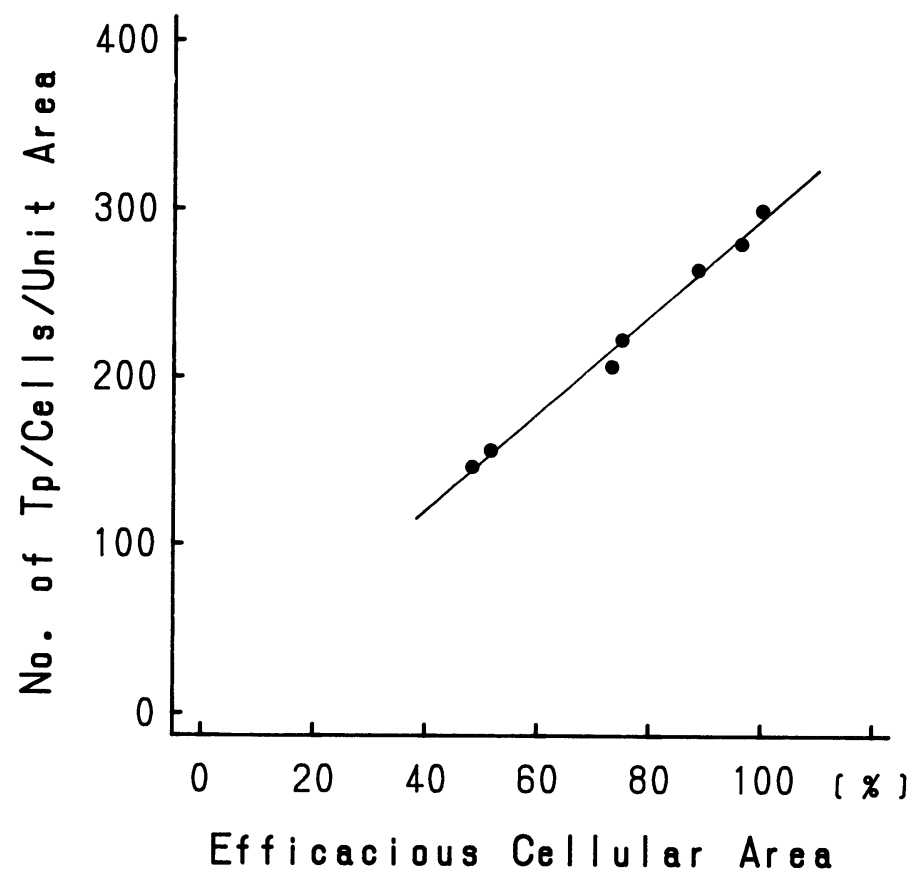

Fig. 2. The relation between the number of intracellular Toxoplasma gondii/UA and the percentage of confluency of the monolayers. The results of seven different experiments were plotted in the figure. Each point represents the mean value of 20 quadrants in two cultures. The correlation is described by the equation, $\mathrm{Y}=2.902 \mathrm{X}+3.864$, with the correlation coefficient $\gamma=0.996$. 


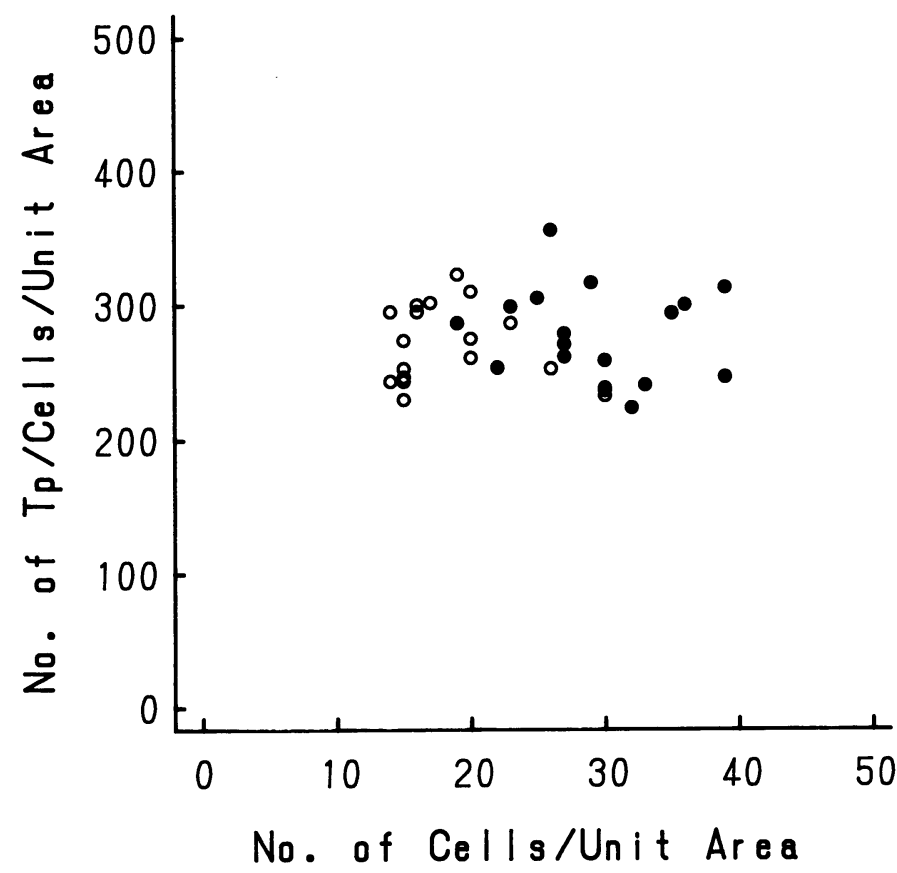

Fig. 3. Correlation between the number of intracellular Toxoplasma gondii/UA and the number of host cells/UA. The numbers of intracellular toxoplasmas/UA of monolayers of two different batches at a constant degree of confluency (approximately 90\%) were plotted against the number of host cells/UA. The relationship between the two values shows nonlinearity as in Fig. 1. The number of intracellular toxoplasmas/UA calculated in either experiment was reasonably reproducible, namely one was $269.0 \pm 40.9$ and the other $264.0 \pm 33.4$.

ranging from 35.4 to $38.1 \%$ with the average of $37.2 \pm 0.9$ (Table I). Comparable results were obtained from the experiments using BRK and BMK monolayer cells.

The numbers of intracellular toxoplasmas/UA from different batches of monolayers having almost the same degree of confluency (approximately 90\%) are plotted in Fig. 3. The mean number of intracellular toxoplasmas/UA was again demonstrated to be independent of the number of host cells/UA as shown in Fig. 1. The mean number of intracellular toxoplasmas/UA calculated in either experiment is reasonably reproducible, namely one is $269.0 \pm 40.9$ and the other $264.0 \pm 33.4$.

These findings indicate that the trophozoites, once they contact the host cells, do not move a long distance to find suitable cells to parasitize and enter almost 
instantaneously the cells where they attach, resulting in such a number of intracellular toxoplasmas/UA proportional to the area occupied by the cells.

Relationship between the Number of Intracellular Toxoplasmas/UA and the Concentration of Toxoplasmas Inoculated

Finally, it was assumed that the number of intracellular toxoplasmas/UA would increase with increasing concentrations of toxoplasmas inoculated at a constant confluency of monolayer.

Toxoplasmas suspensions at various concentrations were overlayed onto monolayers with percentage of confluency of approximately $52 \%$ (Fig. 4).

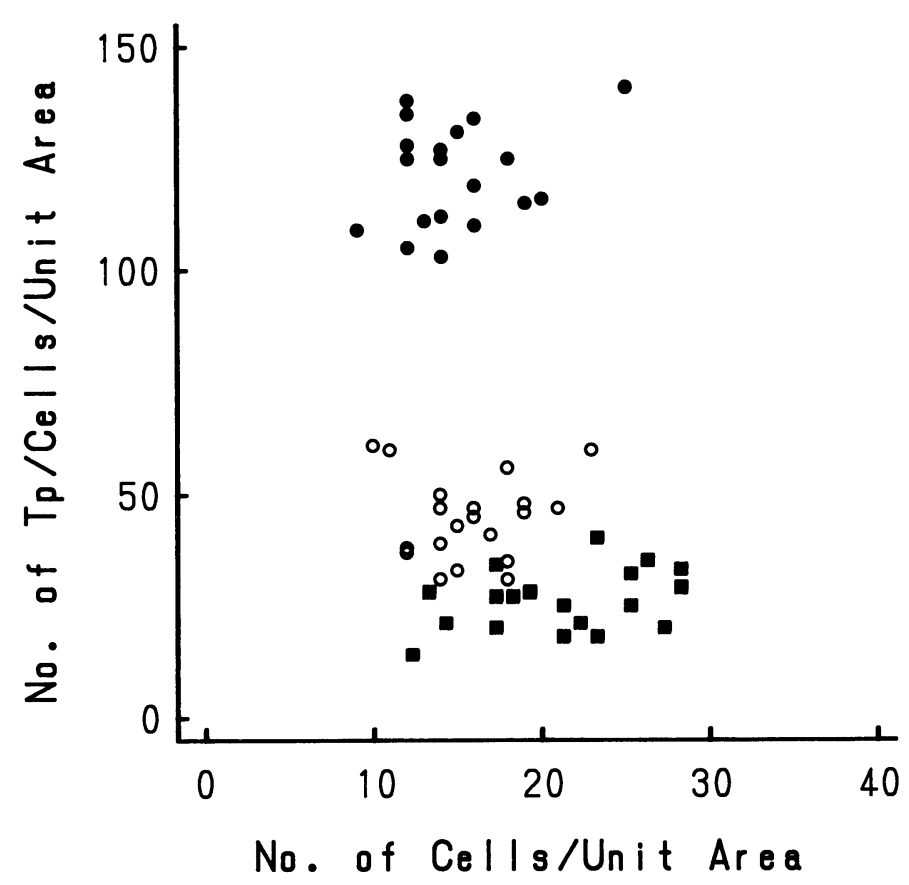

Fig. 4. Correlation between the number of intracellular Toxoplasma gondii/UA and that of host cells/UA with various inoculation sizes of toxoplasmas. Monolayer cultures from the same batch, of which percentage confluency was approximately $52 \%$, were infected with $T$. gondii at concentrations of $\bigcirc: 2.5 \times 107,1.0 \times \bigcirc: 107$, and $\mathbf{a}: 0.5 \times 10^{7}$ cells/ $60-\mathrm{mm}$ dish. The correlation coefficients obtained were 0.261 , 0.042 and 0.339 , respectively. 


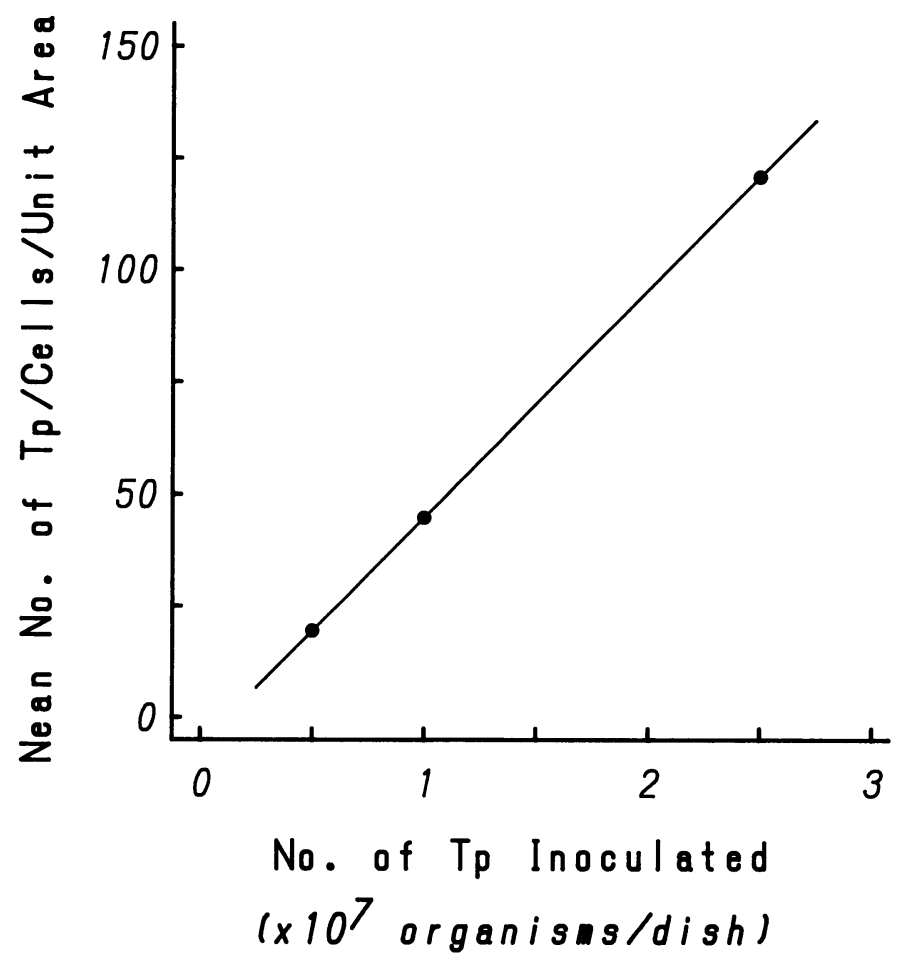

Fig. 5. The relation between the number of intracellular Toxoplasma gondii/UA and the concentration of toxoplasmas inoculated. The number of intracellular toxoplasmas/UA was plotted against the concentration of toxoplasmas inoculated. The figure corresponds to Fig. 4. Regression analysis indicated a slope of 50.654 , intercept $=-5.838$ and $\gamma=1.00$, demonstrating linear dose-dependent relationship between the two values.

Intracellular toxoplasmas/UA increased in number with increasing concentration of toxoplasmas inoculated. The number of intracellular toxoplasmas/UA was found to be constant at each inoculum size of toxoplasmas regardless of the number of host cells/UA. The line drawn in Fig. 5 clearly demonstrates that the relationship between the number of intracellular toxoplasmas/UA and toxoplasmas inoculated is proportionate $(\gamma=1.00)$. 


\section{DISCUSSION}

A method for titration of the infectivity of Toxoplasma gondii in cell cultures was first developed by Chaparas and Schlesinger (1), who applied the plaque counting method of Dulbecco and Vogt (2) to this quantitative determination. This method, however, takes at least 4 to 5 days, and sometimes 12 to 14 days, to obtain titers $(1,8)$. It can be adopted only for low-level infections of monolayers with the parasite. If single host cells contain more than two toxoplasmas, each parasite is not represented by an individual plaque in the monolayer. Thus, the number of intracellular toxoplasmas cannot be directed by the number of plaques. Lycke and Lund $(3,9)$ developed a tissue culture method for titration of infectivity of this parasite using HeLa cells as host cells. In this method the number of intracellular toxoplasmas and that of host cells observed were counted. The number of host cells per microscope field was used to calculate the total number of host cells per culture. Then, the number of infective toxoplasmas per unit volume of inoculum was calculated from the above values. This method assumes that the number of host cells per culture is proportional to the number of host cells per microscope field. It is thus essential that the cell cultures must be standardized satisfactorily and that any variation in the number of cells must be well known (3).

In the present experiments, it was found that [1] the confluent monolayer provided for equal opportunities for infection by each toxoplasma on the monolayer. [2] The number of intracellular toxoplasmas/UA was proportional to the percentage of confluency at a constant number of toxoplasmas inoculated but did not depend on the number of host cells/UA. This leads to the conclusion that the percentage of confluency of the monolayer is equivalent to that of the efficacious area for toxoplasma infection. [3] Finally, the number of intracellular toxoplasmas was proportional to that of toxoplasmas inoculated at a constant percentage of confluency. From these findings, we developed a method for quantitative determination of the infectivity of toxoplasmas. In this method, the number of intracellular toxoplasmas/UA and the percentage of confluency of the monolayer are measured. The percentage of confluency is used for conversion of the number of intracellular toxoplasmas that had entered per unit area to the percentage of infective toxoplasmas. A linear dose-response relationship with a high accuracy was obtained and on an average approximately $37 \%$ of the inoculated parasites were found to enter the host cells (Table I). This value compares very favorably with the value $40 \%$ reported by Lycke and Lund (3).

This method has several advantages. Results of titrations may be obtained at 
$2 \mathrm{hr}$ after inoculation or at any time thereafter. Any type of cell or cells from different animals, so far as we examined, can be used as host cells. With the use of $50 \%$ or more confluent monolayers, the infectivity of toxoplasma can be measured with greater accuracy and reproducibility. Finally, if confluent monolayers $(100 \%$ confluency) are employed, the process of measuring the percentage of confluency can be omitted, and consequently the calculation for titration of the infectivity will be simplified. This method, however, may not be applicable to titration of infectivity of toxoplasmas for phagocytic cells, such as macrophages, or nonadherent cells. Since these phagocytic cells migrate and phagocytize toxoplasmas actively. Thus, there may be no simple way to measure the efficacious cellular area of this type of cell culture for toxoplasma infection.

\section{ACKNOWLEDGEMENTS}

We wish to express our gratitude to Dr. B. Hammerberg of North Carolina State University for his critical reading and revision of the manuscript and to Dr. S. Hayashi, former Director-General of National Institute of Health, Tokyo, for his interest and helpful discussions. The rats of Wistar Imamichi strain were a kind gift from Dr. K. Suzuki of Nippon Veterinary and Zootechnological College. This study was supported in part by a Grant-in-Aid for Scientific Research (63570186) from Japanese Ministry of Education, Science and Culture.

\section{REFERENCES}

1. Chaparas, S. D. and Schlesinger, R. W. (1959): Proc. Soc. Exptl. Biol. Med., 102, 431-437.

2. Dulbecco, R. and Vogt, M. (1954): J. Exptl. Med., 99, 167-182.

3. Lycke, E. and Lund, E. (1964): Acta Pathol. Microbiol. Scand., 60, 221-233.

4. Ahronheim, G. A. (1979): Proc. Soc. Exptl. Biol. Med., 161, 522-526.

5. Kaufman, H. E., Remington, J. S. and Jacobs, L. (1958): J. Ophthalmol., 46, 255-261.

6. Ryning, F. W. and Remington, J. S. (1978): Infect. Immun., 20, 739-743.

7. Endoh, T., Tokuda, H., Yagita, K. and Koyama, T. (1987): J. Protozool., 34, 291-295.

8. Lund, E., Lycke, E. and Sourander, P. (1963): Acta Pathol. Microbiol. Scand., 57, 199-210.

9. Lycke, E. and Lund, E. (1964): Acta Pathol. Microbiol. Scand., 60, 209-220. 